\title{
CLINICAL AND RADIOGRAPHIC EVALUATION OF DEMINERALIZED FREEZE-DRIED BONE ALLOGRAFT PLUS HYALOURONIC ACID VERSUS DEMINERALIZED FREEZE-DRIED BONE ALLOGRAFT PLUS PLATELET-RICH PLASMA FOR THE TREATMENT OF PERIODONTAL INTRABONY DEFECTS - A RANDOMIZED BLIND STUDY
}

\author{
Naglaa M. El-Wakeel*
}

\begin{abstract}
Objectives: The present study aimed to evaluate the effect of ; 1- combined demineralized freez dried bone allograft( DFDBA)plus hyaluronic acid(HA) compared to DFDBA alone, and 2) combined DFDBA/HA compared to DFDBA /PRP in treatment of periodontal infrabony defects(PIDs).
\end{abstract}

Subjects and methods:45 infrabony defects in subjects with chronic periodontitis were randomly divided into 3 groups treated with, group 1: DFDBA/HA, group 2: DFDBA/PRP and group 3: DFDBA alone. Clinical and radiographic measurements were made at baseline and 12 months after surgery. ANOVA followed by Tukey's post hoc test were used to analyze the data.

Results: A statistically significant improvement in clinical attachment level( Cal) and pocket depth ( PD) as well as radiographic bone fill were reported in group 1 and 2 compared to DFDBA alone $(\mathrm{p} \leq 0.05)$, a non significant difference was reported between group 1 and group 2 regarding clinical and radiographic parameters $(\mathrm{p}>0.05)$.

Conclusion : Results of the present investigation suggests that the combination of DFDBA/HA is more effective both clinically and radiographically than DFDBA alone in treatment of PIDs and that both HA and PRP combined with DFDBA are equally effective in treatment of these defects.

\section{INTRODUCTION}

Periodontal disease designates inflammation of tooth-supporting tissues, including cementum , periodontal fibers and alveolar bone. The ultimate goal of periodontal treatment is the regeneration of lost periodontal tissues during the disease process. A variety of treatment approaches, including the use of bone grafts and substitute materials, guided tissue regeneration (GTR) with the use of growth factors, and chemotherapeutics have been proposed to promote regeneration of periodontal tissues ${ }^{(1,2)}$.

* Associate professor of Oral Medicine, Diagnosis, Periodontology and Radiology, Faculty of Dentistry, Al- Azhar (Girls Branch) and MSA Universities, Egypt. 
Demineralized freeze-dried bone allograft, has been used alone and in combination with other treatment modalities for periodontal therapy owing to its osteoconductive and osteoinductive potential $^{(3,4)}$. The observations from histological and clinical studies have shown that some of the grafting procedures may result in healing that can be defined as "periodontal regeneration." However, complete and predictable reconstruction of periodontal tissues is still difficult to obtain ${ }^{(5)}$. Critical clinical components for PIDs successful outcomes are: 1- Wound stability during the early healing sequence, 2- Space provision to allow the migration and proliferation of cells from the PDL and alveolar bone and 3- conditions favoring primary intention healing ${ }^{(6)}$.

The healing of periodontal surgery wound is complicated, as different cells and growth factors are involved in the healing process ${ }^{(7)}$. Studies on wound healing have shown that, after surgery, platelets participate in clot formation and release a series of growth factors involved in cell division and differentiation. Consequently in the repair and tissue forming, therefore, these growth factors can be used to enhance tissue healing process $^{(8)}$. Platelet-rich plasma is a volume of plasma fraction of autologous blood having platelet concentrations above baseline. As being rich in growth factors that play an essential role in tissue healing-such as transforming growth factor- $\beta$, vascular endothelial growth factor, and platelet-derived growth factor, PRP is used in various surgical fields to enhance bone and soft-tissue healing by placing supra physiological concentrations of autologous platelets at the site of tissue damage, favorable periodontal regenerative outcomes was reported to be enhanced by the combined use of PRP with bone allografts $^{(9,10)}$.

Hyaluronic acid (HA) is a naturally occurring linear polysaccharide of the extracellular matrix of connective tissue, synovial fluid, and other tissues that possesses various physiological and structural functions. In the field of dentistry, preliminary clinical trials have been conducted by. Pagnacco e al. ${ }^{(11)}$ in 1997. Hyaluronate has shown anti-inflammatory, anti-edematous and anti-bacterial effects for the treatment of gingivitis and periodontitis ${ }^{(12)}$. Due to its acceleration in periodontal tissue healing properties, it was used as an adjunct to mechanical therapy ${ }^{(13)}$, HA administration to periodontal wound sites could achieve comparable beneficial effects in periodontal tissue regeneration and periodontal disease treatment ${ }^{(14)}$,urther, Hakansson et al. suggested a role of HA in migration and adherence of polymorphonuclear leukocytes and macrophages at the inflamed site and the phagocytosis and killing of invading microbes. Such events would allow counteraction of colonization and proliferation of anaerobic pathogenic bacteria in the periodontal tissues ${ }^{(15)}$.HA was reported to accelerate bone regeneration by chemotaxis, proliferation and successive differentiation of mesenchymal cells. HA has been reported to share bone induction characteristics with osteogenic substances such as bone morphogenetic proteins and osteopontin ${ }^{(16)}$.

The effectiveness of PRP in combination with different types of grafting materials (with or without GTR) has been evaluated in regenerative periodontal therapy and mostly PRP is combined with bone grafts in the treatment of intrabony defects, since the space maintenance of the defect is a crucial factor in periodontal regeneration ${ }^{(17,18)}$. Several previous reports have evaluated the clinical and radiographic outcome of the combination of DFDBA + PRP in treatment of infrabony defects, and the majority of these reports proved a positive outcome $^{(18,19)}$, some others failed to prove that PRP + DFDBA is better than DFDBA alone in treatment of infrabony defects ${ }^{(20)}$.

Hyaluronic acid has been proven to be effective in treatment of gingivitis ${ }^{(12)}$, as an adjunct to scaling and root planning ${ }^{(13)}$ and as an adjunct to open flap 
debridement ${ }^{(14)}$. To the best of my knowledge, no data is available on the use of HA plus DFDBA in the treatment of periodontal infrabony defects in humans.

Therefore, the present randomized blind study aimed to clinically and radiographially; 1) Evaluate the use of HA plus DFDBA in treatment of periodontal infrabony defects as compared to DFDBA alone. 2) Compare between the use of HA plus DFDBA versus PRP plus DFDBA in treatment of infrabony periodontal defects 12 months after flap surgery.

\section{MATERIALS AND METHODS}

\section{Study population}

A total of 45 patients with chronic periodontitis (25 males and 20 females) were selected from the outpatient clinic, Department of Oral medicine and Periodontology, Al Azhar and MSA Universities, Egypt.

\section{Patients grouping and study design:}

Subjects were randomly assigned by coin toss into equally distributed three groups in a parallel, blind design. Criteria for inclusion were individuals who were systemically healthy, no contraindications to surgery, no abnormal platelet count, had at least one vertical osseous defects with probing pocket depth of $>5 \mathrm{~mm}$ and clinical attachment loss $<5 \mathrm{~mm}$ along with radiographic evidence of vertical/ angular bone loss in the affected sites and had at least $2 \mathrm{~mm}$ of keratinized gingiva on facial aspect of selected tooth. Criteria for exclusion were smokers, ex-smokers, patients allergic or sensitive to any medication and/or local anesthesia, patients showing unacceptable oral hygiene compliance, pregnant and lactating females and patients with teeth with excessive mobility. The study protocol was explained to the selected patients, informed consent forms were signed prior to treatment.

\section{Initial therapy:}

Each patient received initial therapy which included scaling and root planning using hand and ultrasonic instrumentation, polishing, and oral hygiene instructions prior to surgical therapy. The occlusal adjustment was performed to eliminate centric prematurities and interferences and control mobility.

\section{Clinical parameters:}

Clinical parameters weremeasured preoperatively and at 12 months postsurgery, including pocket depth (PD) measured from gingival margin until depth of the pocket, clinical attachment level(Cal) measured from depth of the pocket to the cementoenamel junction. Measurements were rounded to the highest whole millimeter using the Michigan 0 probe with Williams markings. These measurements were recorded at 6 sites of the selected teeth: Mesiobuccal, mesiolingual, distobuccal, distolingual, midbuccal, and midlingual. Only one measurement per defect, the deepest site of the selected defect at the baseline was included in the result calculations.

\section{Radiographic evaluation:}

Intraoral periapical radiographs (IoRs) were taken by long cone paralleling technique. Before the exposure, individual occlusal acrylic stents were prepared for each patient .Processing and viewing of the X-ray films were standardized . Bone defect depth was measured as the distance from the alveolar crest to the base of the bone defect. The base of defect (BOD) was defined as the most coronal point where the periodontal ligament space showed continuous width. The alveolar crest (AC) level was taken as the crossing of the alveolar crest with the root surface ${ }^{(21)}$. The amount of defect fill was the difference between the initial and final defect depths at recalled time interval. Percentage $(\%)$ of defect fill was the amount of defect fill/baseline defect depth $\times 100^{(22)}$. 


\section{Surgical procedures}

\section{PRP preparation procedures}

Ten milliliters of blood was drawn from each patient by venipuncture of the antecubital vein. Blood was collected into glass tubes containing 10\% trisodium citrate solution as an anticoagulant. The glass tubes containing the blood were centrifuged at $5,600 \mathrm{rpm}$. for $6 \mathrm{~min}$, which resulted in the separation of three basic fractions. Platelet-poor plasma (PPP) was on top of the preparation, PRP in the middle, and the red blood cell (RBC) fraction at the bottom. Two milliliters of the top layer corresponding to PPP were aspirated with a Pasteur pipette and discarded. The PRP was collected in conjunction with the top $1-2 \mathrm{~mm}$ of the RBC fraction, as the latter is also rich in newly synthesized platelets.

\section{Treatment procedures}

A standardized surgical procedure using a conventional periodontal flap surgery was performed by a single operator. After infiltration with local anesthetic, intra-sulcular incisions were made using Bard-Parker surgical blade \#15 and 12 on the buccal and lingual aspects. Full thickness flap was reflected, and alveolar bone in the area of osseous defect was exposed. The osseous defect and inner aspect of the flap were debrided. The root surfaces were planned until a smooth hard consistency was obtained. The defects at test sites were treated and filled using periosteal elevator with HA (Hyadent BG, Bioscience GmBH, Germany) + DFDBA (Dembone, Pacific Coast Tissue Bank, Los Angeles, $C A$ ) (first group), PRP + DFDBA (second group)or DFDBA alone (third group). Surgical flaps were repositioned, sutured with 4-0 silk suture, and periodontal dressing was placed on the surgical area.

\section{Post surgical phase}

Postoperative medications (Amoxycillin 500 $\mathrm{mg}$ thrice daily for 5 days, Metronidazole $250 \mathrm{mg}$ thrice daily for 5 days and Ibuprofen $400 \mathrm{mg}$ twice daily for 5 days) were prescribed. Dressing and silk sutures were removed 1 week postoperatively. Surgical wounds were gently cleansed with $0.12 \%$ chlorhexidine gluconate on a cotton swab. Patients were instructed to rinse with $0.12 \%$ chlorhexidine gluconate during the second postoperative week. All patients were recalled at one-month interval for check-up and evaluation of oral hygiene. The patients were recalled after 12 months following surgical treatment to record the clinical parameters and to take IORs.

\section{Statistical analysis}

Tested values were presented as mean and standard deviation (SD) values. Data were explored for normality using Kolmogorov-Smirnov test of normality. The results of Kolmogorov-Smirnov test indicated that most of data were normally distributed (parametric data), so ANOVA test was used to compare between the three groups, followed by Tukey's post hoc test, when ANOVA revealed a significant difference. The significance level was set at $p \leq 0.05$. Statistical analysis was performed with SPSS 16.0 (Statistical Package for Scientific Studies, SPSS, Inc., Chicago, IL, USA) for Windows.

\section{RESULTS}

Forty -five subjects were included in the study. These were further subdivided into 3 equal groups $(n=15)$, where the bony defects were treated by Bone graft + hyaluronic acid (group 1), bone graft+ PRP (group 2), or bone graft alone (group 3). The average age of patients was $(33.6 \pm 7.23 ; 34.17 \pm 6.5$; $31.8 \pm 6.02$ ) in groups $1,2,3$ respectively, with no statistically significant difference $\mathrm{p}=0.707$ )

\section{Clinical parameters}

At baseline, there was no significant difference between groups regarding all clinical parameters 
$(\mathrm{p}>0.05)$. After 12 months, PD and CAL reduction was greatest in group 3, followed by group 2 and the least values was recorded in Group 3 (DFDBA), with a statistically significant difference $(\mathrm{p}=, 0.021,0.022$ for $\mathrm{PD}$ and CAL respectively) (Table 1, Fig. 1)

\section{Radiographic parameters:}

A greater value of amount of bone fill was recorded in Group 1 (DFDBA + HA), followed by group 2 (DFDBA + PRP), with the least value recorded in group 3 (DFDBA), with a statistically significant difference $(\mathrm{p}=0.001)$, (Table 2$)$

A greater value of percent of bone fill was recorded in group 1 (DFDBA + HA), followed by group 2 (DFDBA + PRP), with the least value recorded in group 3 (DFDBA), with a statistically significant difference ( $p=0.01$ ), (Table 2, Fig. 2)
TABLE (2) Radiographic parameters and significance of the difference between groups (ANOVA test)

\begin{tabular}{|c|c|c|c|c|}
\hline & $\begin{array}{c}\text { Group } 1 \\
\text { (DFDBA+HA) }\end{array}$ & $\begin{array}{c}\text { Group } 2 \\
\text { (DFDBA+PRP) }\end{array}$ & $\begin{array}{c}\text { Group } 3 \\
\text { (DFDBA) }\end{array}$ \\
\hline \multirow{6}{*}{ 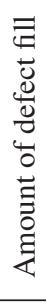 } & Mean & $3.16^{\mathrm{a}}$ & $2.64^{\mathrm{b}}$ & $1.65^{\mathrm{c}}$ \\
\hline & SD & 1.22 & 0.52 & 0.44 \\
\hline & Min & 1.10 & 2.1 & 1.20 \\
\hline & Max & 4.10 & 3.2 & 2.30 \\
\hline & $\mathrm{F}$ value & \multicolumn{3}{|c|}{9.042} \\
\hline & $P$ value & \multicolumn{3}{|c|}{$0.001 *$} \\
\hline \multirow{6}{*}{ 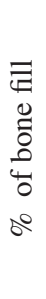 } & Mean & $40.70^{\mathrm{a}}$ & $38.58^{\mathrm{a}}$ & $32.05^{\mathrm{b}}$ \\
\hline & SD & 6.54 & 6.69 & 5.23 \\
\hline & Min & 11.70 & 30.8 & 20.00 \\
\hline & Max & 53.50 & 55.1 & 50.00 \\
\hline & $F$ value & \multicolumn{3}{|c|}{5.514} \\
\hline & $P$ value & \multicolumn{3}{|c|}{$0.01 *$} \\
\hline
\end{tabular}

Significance level $p<0.05$, *significant. Tukey's post hoc test: means sharing the same superscript letter are non significantly different

TABLE (1) Clinical parameters at baseline and after 12 months, percent of each parameter and significance of the difference between groups (ANOVA test)

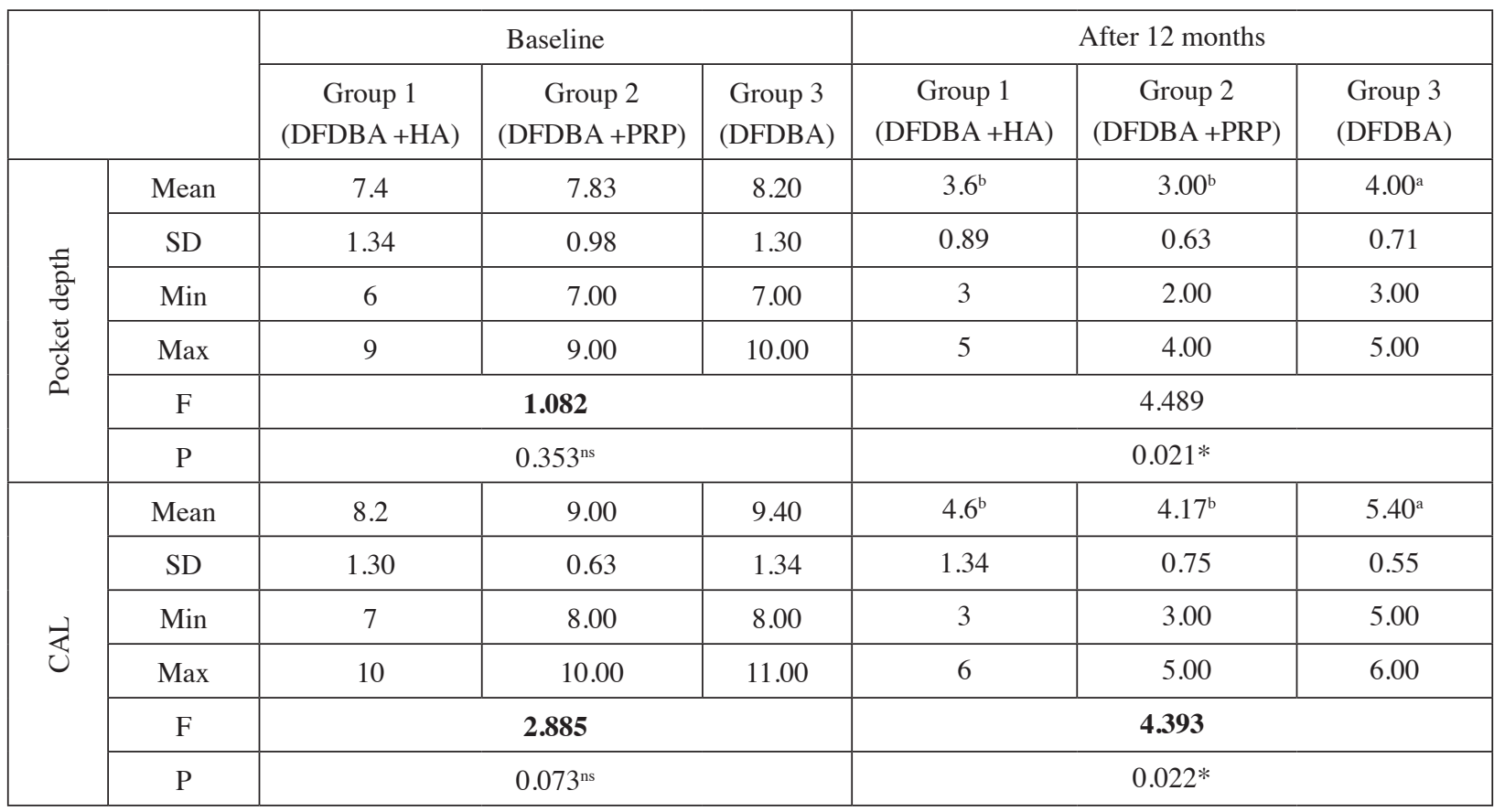

Significance level $p<0.05$, *significant, $n s=n o n$-significant .Tukey's post hoc test: means sharing the same superscript letter are non significantly different 


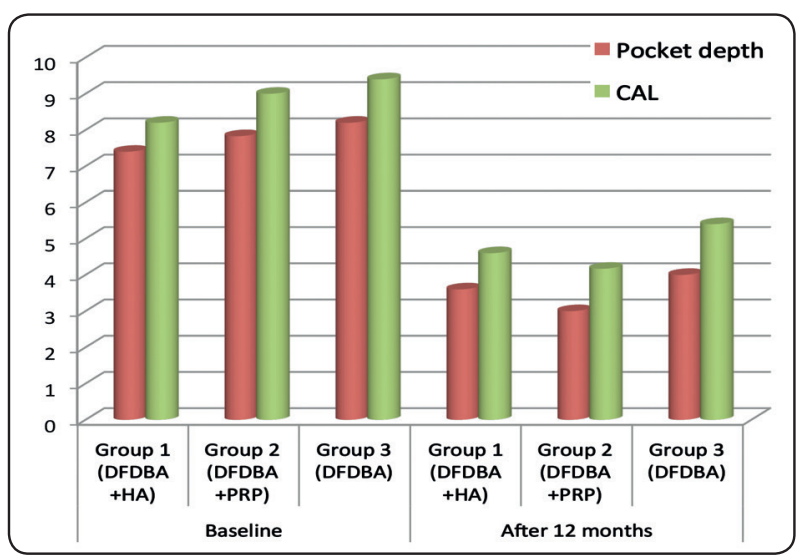

Fig. (1) Column chart showing mean values of clinical parameters at baseline and after 12 months.

\section{DISCUSSION}

The ultimate goal of periodontal treatment is the regeneration of the supporting tissues lost due to periodontal disease. However, In periodontitis, as destruction reaches the deeper structures, regeneration becomes unpredictable ${ }^{(23)}$. The widespread use of DFDBA is based on its osteoconductive and osteoinductive ability ${ }^{(1,3)}$. The present study showed a statistically significant improvement in all clinical and radiographic parameters in the DFDBA group 12 months after therapy compared to baseline data, in agreement with previous studies ${ }^{(1,3,4)}$. The clinical benefits of a regenerative approach employing a biologic mediator combined with bone allografts was reported, based on the assumption that two distinct wound-healing principles may be applied together in one clinical situation ${ }^{(1)}$.The addition of PRP to graft material can serve both in homeostasis and adhesion of graft material, as well as contribute physiologically to more rapid healing of the surgical site $^{(24)}$. As well, Hyaluronan application proved to be promising in wound healing in a number of medical disciplines ${ }^{(25)}$. Thus, their application with conjunction DFDBA in periodontal surgery is thought to achieve additional beneficial effects.

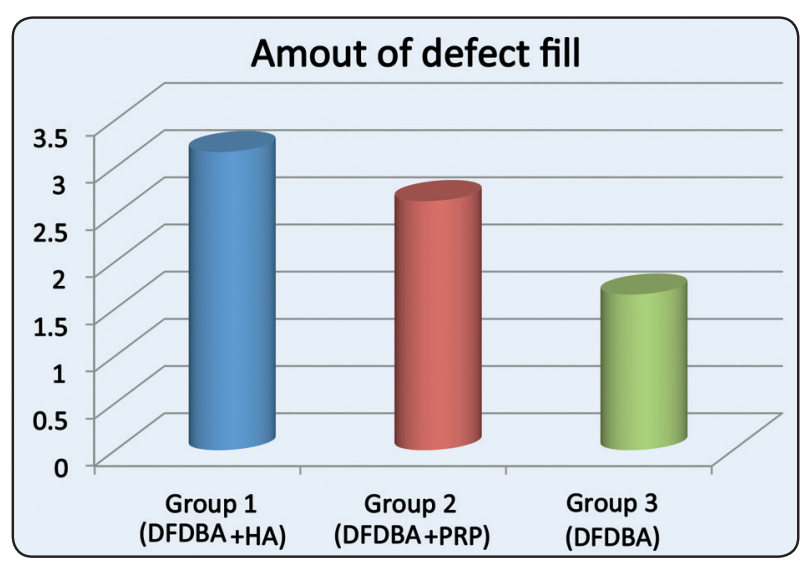

Fig. (2) Column chart showing mean values of radiographic parameters in different groups.

Previous studies investigated the adjunctive effect of hyaluronan application to SRP and to modified Widman flap in the treatment of chronic periodontitis ${ }^{(13,14)}$. It was suggested that HA has osteoinductive potential and accelerates new bone formation during bone wound healing by stimulating osteogenic differentiation, thus might have clinical applications with and without bone graft material(26). However, Hunt et al. ${ }^{(27)}$ demonstrated that HA stimulated bone healing but did not have any osteo- conductive effect, this consequently, the present study investigated the efficacy of using Ha combined with DFDBA in treatment of PIDs compared to DFDBA alone and not HA alone, as a space maintaining effect of the bone graft is needed in treatment of infrabony defects.

This study is the first to report a statistically significant improvement in clinical and radiographic parameters 12 months after surgery compared to baseline data in PIDs treated with HA plus DFDBA. Moreover, this group was statistically significantly better regarding all tested parameters compared to DFDBA alone. The present study reported a statistical improvement in CAL after HA/DFDBA application, this is in agreement with EL-Sayed et al., who reported a significant improvement in CAL after HA application with $\mathrm{MWF}^{(14)}$, and with 
Ballini et al., who reported a positive clinical and radiographic outcome from $\mathrm{HA}$ combined with autologous graft in treatment of infrabony defects ${ }^{(28)}$, but, in contrast to earlier data ${ }^{(13,29)}$ that found a non significant difference in CAL between sites receiving hyaluronan as an adjunct to SRP and sites receiving SRP alone. This is also in contrast to the study of Engström et $\mathrm{al}^{(30)}$, who found no difference in clinical measures between sites treated by guided tissue regeneration (GTR) with hyaluronan and control sites treated with GTR alone.

In this study, a statistical significant reduction of the PD was reported in group 1 compared to group 3 this is in agreement with Erick etal., ${ }^{(31)}$ but in contrast to others who reported a non statistical reduction of PD between tested groups ${ }^{(13,14,30)}$. Variations in hyaluronan gel formulations employed in the different studies, treatment modalities and surgical techniques, disease severity, and follow-up intervals may explain the differences in the results between these studies and the present one.

As far as known, no data are available on radiographic assessment of PIBs bone fill with $\mathrm{Ha} /$ DFDBA, this work demonstrated a significant improvement in bone fill and \% of bone fill in group 1 compared to group 3, this is in agreement with Ballini et al ${ }^{(28)}$, and Sagliyan et al., who investigated the effects of autologous cancellous bone graft plus hyaluronic acid on the healing of experimentally induced bone defects, they demonstrated higher scores in terms of bone healing criteria when graft material combined with HA was used compared to controls ${ }^{(32)}$. These results could be justified by previous observations that pointed out to the assumption that HA can be an ideal vector for the bone morphogenic proteins ${ }^{27,33)}$, induces osteoblast differentiation and bone formation, leading to the suggestion that HA can enhance the osteogenic and osteoinductive properties of bone graft materials and substitutes ${ }^{(34)}$.
This study further confirmed the favorable outcomes of DFDBA/PRP combination in the treatment of PIBDs, a statistically significant improvement in CAL and PD was reported in group 2 compared to group 3. Our data are in accordance with previous studies ${ }^{(1,10,17,18,19)}$, this is explained by additional biologic effects of PRP, Wikesjo et al. have stated that because of its high fibrin content, PRP preparation has a 'sticky' characteristic that works as a hemostatic and stabilizing agent, thus, it may aid in immobilizing the blood clot and bone graft in defect area, which is an important event in early phases of wound healing in periodontal regeneration ${ }^{(35)}$. Our results are is in accordance with other investigators who suggested that PRP is successful as a matrix enhancement factor with graft particles in combination therapies; however, a limited amount of healing is observed when it is used alone ${ }^{(19,24,36)}$. This observation is in agreement with others like Markou et al. ${ }^{(20)}$, who reported a non significant difference between DFDBA /PRP compared to control.

The amount of radiographic bone fill reported in the present work was statistically greater in group 2 compared to group 1 .These results are in accordance with a study by Piemontese et al. and Kukreja etal. ${ }^{(1,10)}$. However, studies by Okuda et al., Aghaloo et al. and Wiltfang et al. showed contrasting results ${ }^{(17,36,37)}$, the limited number of cases in the present work and the morphology of the IBDs could cause the conflicting results . Further, it was suggested that growth factors present in high concentrations at inappropriate times or for an extended duration can adversely affect cell behavior $^{(1,37)}$.

Finally, the present study showed a non significant difference between group 1 and 2 regarding clinical and radiographic parameters. Considering that the use of PRP is more economically suitable to some patient and equally effective as HA in treatment of PIDs, a flexible decision regarding treatment options could be made. 


\section{CONCLUSIONS}

In conclusion, the results of this study indicate that the HA or PRP combination with DFDBA is more effective than DFDBA alone in treatment of PIDs and that both combinations are not statistically different regarding pocket depth and clinical attachment loss reduction as well as radiographic bone fill in treatment of such defects. Further studies with a larger sample size, investigating other parameters such as bacteriological and biomarkers assessment, as well as evaluation the effect of IBD morphology on the outcome are required.

\section{REFERENCES}

1- Kukreja B, Dodwad V, Kukreja P, Ahuja S, Mehra P. comparative evaluation of platelet-rich plasma in combination with demineralized freeze-dried bone allograft and DFDBA alone in the treatment of periodontal intrabony defects: A clinicoradiographic study. J Indian Society of Periodontology 2014; 18:618.

2- Kenney EB. Platelet-rich plasma and bovine porous bone mineralcombined with guided tissue regeneration in the treatment of intrabony defects in humans. J Periodontal Res 2002;37:300.

3- Schwartz Z, Mellonig JT, Carnes DL Jr, de la Fontaine J, Cochra DL, Dean DD, Boyan BD .Ability of commercial demineralized freeze-dried bone allograft to induce new bone formation. J Periodontol 1996;67:918.

4- Quintero G, Mellonig JT, Gambill V, Pelleu G .A six month clinical evaluation of decalcified freeze-dried bone allografts in periodontal osseous defects. J Periodontol 1982; 53:726.

5- Yukna RA, Mellonig JT .Histologic evaluation of periodontal healing in humans following regenerative therapy with enamel matrix derivative. A10 case series. J Periodontol 2000;71:752-759.

6- Sehdev B, Bhongade M,1 and Ganji. K Evaluation of effectiveness of hyaluronic acid in combination with bioresorbable membrane (poly lactic acid-poly glycolic acid) for the treatment of infrabony defects in humans: A clinical and radiographic study. J Indian Soc Periodontol 2016; 20 : 50 .

7- Cortellini , P. \& Tonetti, M. Focus on intrabony defects: guided tissue regeneration. Periodontology 2000 2000; 22:104-132.
8- Creeper, F.; Lichanska, A.M.; Marshall, R.I.; Seymour, G.J. \& Ivanovski, S.. The effect of platelet-rich plasma on osteoblast and periodontal ligament cell migration and proliferation and differentiation. Journal of Periodontal Research 2009;44: 258.

9- Ilgenli T, Dündar N and Kal D. Demineralized freezedried bone allograft and platelet-rich plasma vs plateletrich plasma alone in infrabony defects: a clinical and radiographic evaluation. Clin Oral Invest 2007; 11:51.

10- Piemontese M, Aspriello SD, Rubini C, Ferrante L and Procaccini M. Treatment of periodontal intrabony defects with demineralized freeze-dried bone allograft in combination with platelet-rich plasma: a comparative clinical trial. J periodontal $2008 ; 79: 802$.

11- Pagnacco A, Vangelisti R, Erra C, Poma A. Double-blind clinical trial versus placebo of a new sodium-hyaluronatebased gingival gel. Attual Ter In. 1997;15:1.

12- Pistorius A, Martin M, Willershausen B, Rockmann P. The clinical application of hyaluronic acid in gingivitis therapy. Quintessence Int. 2005;36:531.

13. Xu Y, Höfling K, Fimmers R, Frentzen M, Jervøe-Storm PM. Clinical and microbiological effects of topical subgingival application of hyaluronic acid gel adjunctive to scaling and root planing in the treatment of chronic periodontitis. J Periodontol. 2004;75:1114.

14- El-Sayed K, Dahaba M, Aboul-Ela S, Darhous M. Local application of hyaluronan gel in conjunction with periodontal surgery: A randomized controlled trial. Clin Oral Investig. 2012; 16:1229.

15- Håkansson L, Hällgren R, Venge P. Regulation of granulocyte function by hyaluronic acid. In vitro and in vivo effects on phagocytosis, locomotion, and metabolism. J Clin Invest. $1980 ; 66: 298-305$.

16- Mendes RM, Silva GA, Lima MF, Calliari MV, Almeida AP, Alves JB, et al. Sodium hyaluronate accelerates the healing process in tooth sockets of rats. Arch Oral Biol. 2008; 53:1155-62

17- Okuda, K.; Tai, H.; Tanabe, K.; Suzuki, H.; etal., Plateletrich plasma combined with a porous hydroxyapatitegraft for the treatment of intrabony periodontal defects in humans: a comparative controlled clinical study. Journal of Periodontology,2005;76:890-

18- Agarwal A, Gupta ND, Jain A .Platelet rich fibrin combined with decalcified freeze-dried bone allograft for the treatment of human intrabony periodontal defects: a randomized split mouth clinical trail .Acta odontol Scand 2016;74:36. 
19- Camargo, P.M.; Lekovic, V.; Weinlaender, M.; DivnicResnik, T.; Pavlovic, M. \& Kenney, E.B. A surgical reentry study on the influence of platelet-rich plasma in enhancing the regenerative effects of bovine porous bone mineral and guided tissue regeneration in the treatment of intrabony defects in humans. Journal of Periodontology, 2009;80:.915.

20- Markou N1, Pepelassi E, Vavouraki H, et al., Treatment of periodontal endosseous defects with platelet-rich plasma alone or in combination with demineralized freeze-dried bone allograft: a comparative clinical trial. J periodontal $2009 ; 80: 1911$.

21- Benn DK. A computer-assisted method for making linear radiographic measurements using stored regions of interest. J Clin Periodontol 1992;19: 441.

22- Subbaiah R, Thomas B. Efficacy of a bioactive alloplast, in the treatment of human periodontal osseous defects-a clinical study. Med Oral Patol Oral Cir Bucal 2011;16:e239-44.

23- Wang HL, Greenwell H, Fiorellini J, Giannobile W, Offenbacher S, etal., Periodontal regeneration. J Periodontol 2005; 76:1601.

24- Lekovic V, Camargo PM, Weinlaender M, Vasilic N, Kenney EB.Comparison of platelet-rich plasma, bovine porous bone mineral and guided tissue regeneration versus platelet-rich plasma and bovine porous bone mineral in the treatment of intrabony defects :A reentry study. J Periodontol 2002;73:198-205.

25- Pilloni A, Rimondini L, De Luca M, Bernard GW .Effect of hyaluronan on calcification-nodule formation from human periodontal ligament cell culture. J Appl Biomater Biomech $2003 ; 1: 84$.

26- Moseley R1, Waddington RJ, Embery G., Hyaluronan and its role in periodontal wound healing. Dent Update. 2002;29:144-8.

27- Hunt DR, Jovanovic SA, Wikesjö UM, Wozney JM, Bernard GW. Hyaluronan supports recombinant human bone morphogenetic protein-2 induced bone reconstruction of advanced alveolar ridge defects in dogs. A pilot study. J Periodontol. 2001;72:651-8.
28- Ballini A, Cantore S, Capodiferro S, Grassi F. Esterified Hyaluronic Acid and Autologous Bone in the Surgical Correction of the Infra-Bone Defects. Int. J. Med. Sci. 2009; 6:65.

29- Johannsen A, Tellefsen M, Wikesjo U, Johannsen G. Local delivery of hyaluronan as an adjunct to scaling and root planing in the treatment of chronic periodontitis. J Periodontol $2009 ; 80: 1493$.

30- Engström PE, Shi XQ, Tronje G, Larsson A, Welander U, Frithiof L, Engstrom GN. The effect of hyaluronan on bone and soft tissue and immune response in wound healing. $\mathrm{J}$ Periodontol 2001;72:1192.

31- Eick S, Renatus A, Heinicke M, Pfister W, Stratul S, Jentsch H. Hyaluronic Acid as an Adjunct After Scaling and Root Planing - A Prospective Randomized Clinical Trial. j periodontal 2012;48:941.

32- Sagliyan A ,Hani M, Karabuluti E, Özkaraca M. Research of the effects of autologous cancellous bone graft and hyaluronic acid on the healing of bone defects experimentally induced in rabbits. Turk J Vet Anim Sci 2016;40: 374.

33- Kim D, Valentini F. Retention and activity of BMP-2 in hyaluronic acid-based scaffolds in vitro. J Biomed Mater Res 2002. 59: 573.

34- Huang L, Cheng Y, Koo L, Lee M, Qin L, Cheng C,etal., The effect of hyaluronan on osteoblast proliferation and differentiation in rat calvarial-derived cell cultures. J Biomed Mater Res. 2003; 15:880.

35- Wikesjö M, Nilvexus E, Selvig A. Significance of early healing events on periodontal repair: A review. J Periodontol 1992;63:158-65.

36- Aghaloo TL, Moy PK, Freymiller EG .Investigation of platelet-rich plasma in rabbit cranial defects: a pilot study. J Oral Maxillofac Surg 2002; 60:1176.

37- Wiltfang J,Kloss R, Kessler P, Nkenke E, Schultze-Mosgau $\mathrm{S}$, Zimmermann R, et al. Effects of platelet-rich plasma on bone healing in combination with autogenous bone and bone substitutes in critical size defects. An animal experiment. Clin Oral Implants Res 2004;15:187. 